\title{
El control de la construcción y el control urbano en Colombia: El caso Medellín y la Ruta Medellín Vivienda Segura 2014-2024; retos y oportunidades
}

\author{
Controls on Building and the Urban Environment in Colombia: Medellin and the Route to Safe \\ Housing 2014-2024; Challenges and Opportunities
}

\author{
Diego Restrepo Isaza (1) \\ (1) Especialista en Derecho inmobiliario, Universidad Pontifica Bolivariana, Medellín, Colombia. Consejero Alcaldía de Medellín, Programa \\ Gerencia de Vivienda Segura, Medellín, Colombia.diego.restrepoi@medellin.gov.co; drisaza@gmail.com; @drisaza
}

Recibido 8 de septiembre de 2014. Modificado 13 de febrero de 2015. Aprobado 17 de febrero de 2015.

DOI: http://dx.doi.org/10.16924/riua.v0i41.789

\section{Palabras clave \\ Construcción, control urbano, curadores, edificio Space, licencias, Medellín, supervisión, vivienda segura. \\ Resumen \\ La Alcaldía de Medellín creó el programa de Vivienda Se- gura en 2014. Como consejero de esta Alcaldía, compartiré en este artículo algunos hallazgos en temas de normativa de construcción en Colombia, dificultades y aciertos con leyes y curadurías. Además, daré algunas recomendaciones sobre lo que se debe hacer para mejorar.}

\section{Key words}

Building, urban control, development officials, Space building, licenses, Medellin, supervision, safe housing.

\section{Abstract}

The Mayor's Office of Medellin created the Department of Safe Housing in 2014. As a consultant of this Office, I share in this article some findings on the subjects of building regulations in Colombia, as well as difficulties and successes with laws and departments of urban development. I also offer some suggestions for improvements.

\section{INTRODUCCIÓN}

En este artículo hablaré sobre lo que le costó a Medellín, y a lo que nos ha llevado el caso del colapso de la torre 6 de la Urbanización Space. La ciudad tiene cerca de 2'400.000 de habitantes, de los cuales el 75\% es de estratos 1, 2 y 3, es decir, estrato bajo y estrato muy bajo. Esta condición particular de la ciudad, trasladado al tema de la construcción, evidencia la presencia de una alta informalidad en su crecimiento. La mayoría de nuestras ciudades latinoamericanas están sometidas a procesos de informalidad en muchos sectores económicos, que han tratado de regularse con el fin de formalizar aquellas situaciones de hecho, lo cual no es ajeno a lo que sucede en el sector de la construcción, donde la mayoría de las normatividades están diseñadas para ser aplicadas al sector formal, pero no para el sector informal. Este es un tema que debería preocuparnos, porque necesitamos encontrar una solución que se adecúe a esta condición de informalidad de muchos de nuestros edificios, que en los barrios populares de Medellín alcanzan más de seis pisos construidos de manera informal. Muchas de estas construcciones se generaron en procesos de legalización y creación de suelo como el Plan Terrazas (plan promovido por el gobierno municipal en los años setenta y ochenta, que permitía al poseedor de una vivienda de una planta construir un segundo piso con incentivos económicos).

Sin embargo, Space no fue un caso presentado en la construcción informal de nuestra ciudad; se trata de una edificación ubicada en la comuna con mayor calidad y nivel de vida y, mayores ingresos económicos de nuestra ciudad, El Poblado.

\section{SPACE: UN CASO AISLADO ¿O NO?}

En el periódico Portafolio se publicó el 16 de octubre de 2013 una noticia titulada "El desplome del edificio Space es un caso aislado", justo tres o cuatro

${ }^{1}$ Este artículo es el resultado de la ponencia presentada por Diego Restrepo Isaza en el foro "Los códigos en la construcción en Colombia: Aproximaciones y Aplicaciones", realizado en la Universidad de los Andes el 8 de septiembre de 2014. 
días después de la tragedia. Ahí el gremio de la construcción de nuestro país ya estaba calificando el fenómeno de la caída del edificio como un caso "aislado" solo tres días después. Sin embargo, y a medida que han avanzado las investigaciones, y que aún hoy continúan, se evidenció que el problema no es solo de una edificación, de una constructora, o de un barrio de nuestra ciudad. Se reveló que las fallas de construcción en Colombia obedecen a un problema sistémico como lo pretendemos demostrar en el presente escrito, para lo cual nos apoyamos en casos como el del Barrio Campo Alegre en Barranquilla, donde los medios informan sobre más de 10.000 viviendas construidas y que presentan reclamaciones por daños originados en el suelo.

En la Figura 1 se presenta la caracterización socioeconómica de los compradores afectados por CDO, señalando que el $85 \%$ de los afectados pertenece
Si bien la vida debe ser protegida en toda su integridad, se debería también proteger el patrimonio y garantizar el derecho a la vivienda; de igual manera, proteger el patrimonio público de los municipios, pues las responsabilidades de control de construcción, que no es responsabilidad de los municipios sino de los propietarios de la obra, hacen que el afectado de un siniestro le reclame al Estado, en este caso a los municipios, por su presunta omisión en la pérdida patrimonial sufrida ante la insolvencia del constructor, confundiendo control urbano y control de construcción.

Hoy tenemos 4.000 viviendas afectadas por los diseños irresponsables de Jorge Aristizábal Ochoa, ingeniero muy reconocido por sus métodos innovadores de construcción sismo resistente. En la Gerencia de Vivienda Segura tenemos un listado de 7.000 viviendas más por revisar que él también construyó.

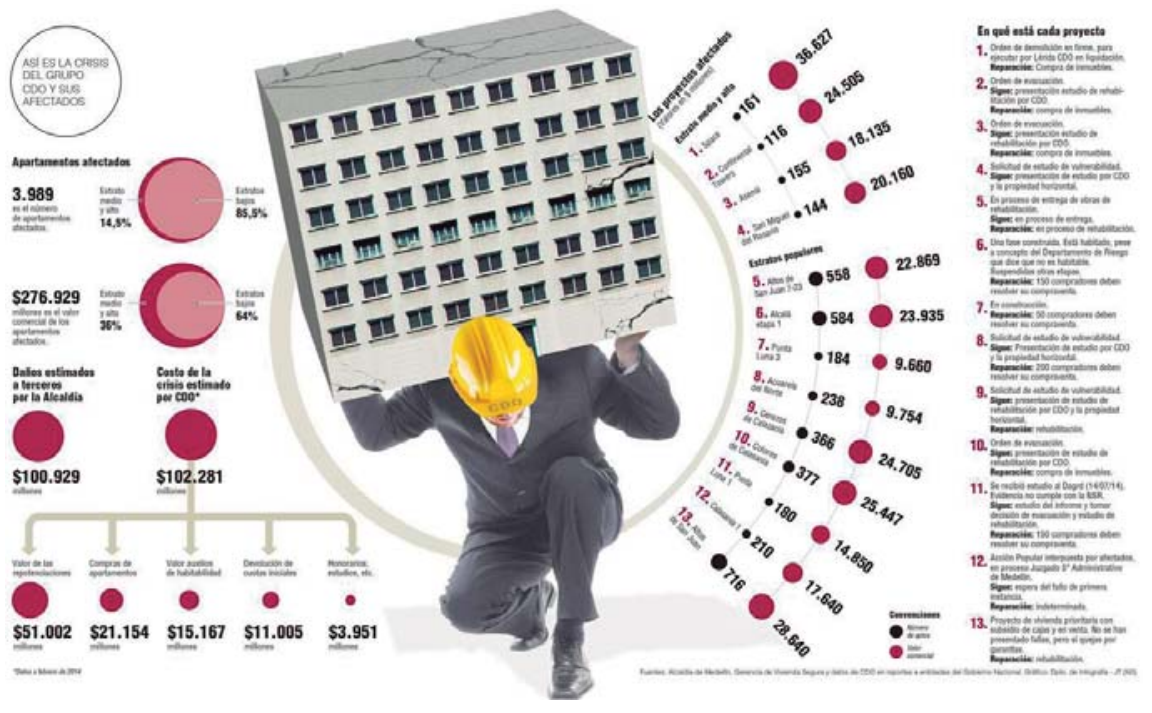

Figura 1. Descripción de los afectados por CDO. Fuente: Alcaldía de Medellín, 2014.

al estrato socioeconómico 3, es decir, estrato medio. Las familias afectadas por Space, en su gran mayoría, están conformadas por jóvenes profesionales, que empiezan a formar un patrimonio, y que invirtieron todos sus ahorros en su primer apartamento. A ellos, el sistema no los protegió, como en el caso de Barranquilla.
Planeación, la nueva sede del banco en Rionegro fue desalojada a principios de mayo”. Esta decisión se tomó una vez se revisaron los diseños estructurales de la construcción elaborados por el ingeniero Aristizábal. En este momento esa edificación está en proceso de rehabilitación.

El caso de la falla en los diseños estructurales no es un tema de construcción en las laderas, porque también en algún momento lo identificaron así. Medellín tiene que adaptarse a su suelo y a un valle angosto; un suelo que tiene sus características negativas, como cualquier otro suelo.

El 1 de septiembre de 2014 la firma Ingeniería Sísmica Estructural SAS, encargada de hacer la investigación sobre el colapso de la torre 6 de Space para la Fiscalía General de la Nación, concluyó lo siguiente: “un diseño estructural inadecuado y violatorio de los principios mínimos de comportamiento estructural que se establecen en la legislación de sismo resistencia nacional; el diseño estructural de la edificación que desconoce y transgrede los aspectos fundamentales del ordenamiento legal vigente que regula el diseño y construcción sismo resistente del país; no se realizó la revisión de las memorias y los planes estructurales ordenada por la ley y, si se realizó, se hizo de una manera negligente y superficial, ya que permitió la aprobación de un proyecto estructural que es transgresor de los elementos fundamentales establecidos por el ordenamiento jurídico legalmente vigente en el país”. Por esto están implicados los dos curadores, el curador que aprobó el Plan de Urbanismo General (PUG) y el que aprobó también la etapa 6 de la edificación. Este concepto fue tan tajante y concluyente que motivó al municipio de Medellín para que se declarara víctima también en el proceso penal, lo cual fue reconocido por el juez penal.

Las causas que incidieron en el colapso, dice el informe, fueron las bajas resistencias de concretos usados en obra en elementos primordiales del sistema de carga vertical que contribuyeron a 
acelerar el mecanismo de colapso progresivo de la edificación. Se puede ver que, según el estudio, no era solamente un tema de diseño, era también un tema de construcción. Igualmente, se señala la respuesta negligente del diseñador estructural ante los avisos de sobre esfuerzos que se presentaban en la edificación; inexistencia de una supervisión técnica adecuada del proyecto para los productos de este accionar irracional y temerario y más elementales de comportamiento; un diseño estructural con transgresiones y un desconocimiento reiterativo al ordenamiento legal vigente.

\section{UNA FALLA DEL SISTEMA}

En nuestra función, tenemos contacto permanente con la firma CDO y una de las observaciones que nos hacen permanentemente es que ellos también son víctimas del sistema; ellos estaban muy tranquilos porque contaban con el sello de calidad del Icontec, que les permitía ser exonerados de la supervisión técnica independiente, y habían contratado a uno de los mejores ingenieros del país. Uno, como abogado (profesión que llevo con orgullo y que fue la primera profesión que existió en la humanidad, porque todo al principio era un caos) empieza a encontrarse con la letra menuda y concluye que este conflicto hoy es un tema de letra menuda. Cualquier curador, cualquier ingeniero en toda esta cantidad de códigos y normas que permanentemente se publican, tiene la forma de evadir su responsabilidad. Esto refleja lo que hoy tenemos: un problema estructural y sistémico.

Con respecto al control, muchos le achacan responsabilidades a la Alcaldía, lo cual nos ha motivado a que estudiemos en qué consiste el control de una construcción. Se debe identificar la diferencia entre control de construcción y control urbanístico. Hay dos leyes, la ley 388 y la ley 400, ambas expedidas en 1997 pero que no se hablan, al igual que los planes de ordenamiento territorial que tampoco se relacionan con las normas de construcción. Estas dos leyes regulan este impresionante mare magnum normativo. La ley 400 regula el control de construcción al señalar que la supervisión técnica es $100 \%$ independiente, no la hace el municipio. De ahí que el control de construcción esté a cargo del supervisor técnico y de la Comisión Asesora Permanente, organismo que interpreta y da el alcance de la aplicación de las normas de construcción. Por otro lado, está el control urbanístico definido como aquella vigilancia que ejerce el municipio para que las obras se realicen conforme a la licencia otorgada y a los POT, sin que sea necesario volver a revisar la licencia, ni ejercer una actividad propia de interventoría.

Sin embargo, el desconocimiento de estos temas por parte del sector, nos lleva a afirmaciones como las de un curador urbano, él consideraba que el municipio debía revisar los diseños, controlar que la obra se realizara conforme a la NSR y verificar el cumplimiento de las especificaciones técnicas de construcción. ¡Gran error! Se imaginan el avance de una obra en su cronograma esperando la visita del municipio para avanzar en un proyecto. El control de construcción no puede estar a cargo de los municipios, pues ellos no pueden revisar si se cumplió con el número y la calidad del hierro de una construcción, o si el diseño sismo resistente fue bien diseñado y revisado. La ley ya otorga una responsabilidad clara en cuanto a la elaboración, la revisión y la aprobación de diseños y en ningún momento señala que el municipio deba realizar nuevamente esta labor. Olvidémonos de asignarle una responsabilidad al municipio que no es.

El sistema normativo en los procesos de licenciamiento y construccción evidencia que estamos ante un problema sistémico. Por ejemplo, el artículo 56 del decreto 1469 del 2010, que regula el trámite de licencias de construcción, prohíbe que los curadores urbanos exijan el cumplimento de cualquier tipo de normas expedidas por las alcaldías relacionadas con las condiciones de diseño y técnicas de construcción, ya que estas corresponden exclusivamente a la Comisión Asesora. Los municipios en sus códigos de construcción no se han adaptado a esta nueva normatividad ni se han integrado con el trabajo de los curadores. Esto hace que la comunidad en general los califique como ruedas sueltas que hacen y deshacen en la aprobación de licencias, conforme los constructores quieran.

Colombia posee un sistema normativo bien diseñado, pero consideramos que la aplicación del sistema normativo tiene sus fallas, ya sea por falta de una reglamentación técnica al respecto, o por la falta de ética de algunos profesionales. A la Gerencia de Vivienda Segura de la Alcaldía de Medellín nos llegan muchos anónimos (desde hace mes y medio estamos dedicados a este conflicto 24 horas al día), y en uno de tantos se dice que el verdadero culpable es la Comisión Asesora Permanente de Construcciones Sismo Resistentes creada por la Ley 400, al señalar que hay una pasividad de la Comisión en el tema de las reglamentaciones especiales. A manera de ejemplo, y con todo respeto de quienes integran dicha Comisión, en el acta 118 de la reunión efectuada el 8 de noviembre de 2013, primera reunión celebrada luego del colapso del edificio Space, se dice, en el punto número cinco denominado Acreditación profesional, estado actual y plan de acción, lo siguiente: "Se comenta que la Comisión dentro del alcance de las funciones que le atribuye la ley 400 puede definir los procedimientos para llevar los registros de la experiencia de los profesionales. Para ello se propone firmar convenios con la Sociedad Colombiana de Ingenieros y con la Sociedad Colombiana de Arquitectos para que ellos realicen el primer paso del proceso de acreditación que es el de certificar la experiencia y llevar el registro de los profesionales. Se pide elaborar unos términos de referencia para solicitar una propuesta de las dos asociaciones para llevar a cabo este primer paso de la acreditación profesional". Vale la pena reflexionar al respecto, pues es una decisión que se toma 17 


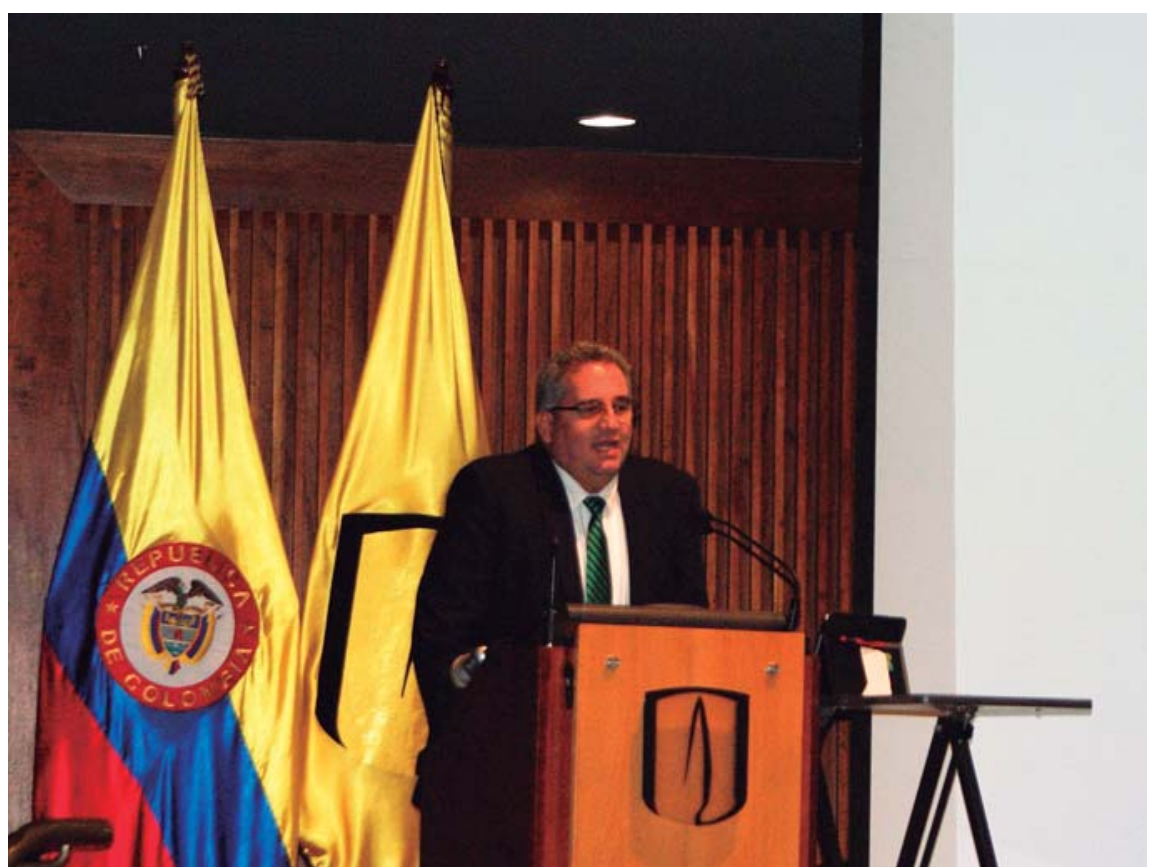

Diego Restrepo Isaza. Fuente: Archivo Particular, Universidad de los Andes

años después de expedida la Ley 400 y en una reunión que se da justo después del colapso de un edificio. Sin embargo, es claro que es importante avanzar en el tema de la certificación laboral para aclarar el campo de acción de estos profesionales, el alcance de la supervisión técnica y la revisión de los diseños que está a cargo de los curadores.

De otro lado, y con el fin de reforzar nuestra teoría de la falla sistémica del sector, este ha entrado en un proceso de desregularización en la última década, que ha permitido pasar de un una participación del 3\% del PIB en los primeros años de la década pasada a una participación cercana al 9\% en el presente año. Esa desregularización en parte ha facilitado ese crecimiento. Creemos que no se trata de crear normas, o derogarlas, sino de ordenar las que ya están para evitar más tragedias. Un ejemplo nos ilustra el tema de la desregularización del sector: Cuando se quieren comprar unas acciones de una sociedad en el mercado de valores, a quien compra le piden una serie de documentos para soportar la venta y no puede ir a comprarlas a cualquier lado; existen unos organismos del sector financiero que las venden, y son vigilados por una Superintendencia. En cambio, si se quiere adquirir un apartamento de uno o dos millones de dólares, es posible comprarlo sin pasar por ningún control (salvo el control cambiario presente en cualquier actividad económica). Cualquier persona puede vender un inmueble sin un control realmente de esta actividad.

\section{UNA PROPUESTA DE REFORMA}

De esta falla sistémica que hemos pretendido demostrar, se desprendió una propuesta. La Alcaldía de Medellín Asociación de Ciudades Capitales, al Ministerio de Vivienda Ciudad y Territorio una solicitud de reforma de algu-

En relación con la regulación de los curadores y las curadurías, no se sabe quién es el curador ni qué es la curaduría, a diferencia del notariado y registro: si a un notario lo declaran insubsistente él tiene que guardar todos los libros de protocolo que hacen parte del estatuto notarial y de la notaría; si a un curador lo destituyen él se puede llevar todas las licencias de construcción para su casa. El sistema de curadurías no presentó, después de un trabajo con la nos puntos: está reglamentado, mientras que la figura del curador urbano sí. Se solicitó reglamentar el tema de la revisión de diseños y crear la superintendencia de control de curadurías y de la actividad constructora e inmobiliaria.

En el tema de las licencias se pidió reglamentar los términos de estas, para controlar que el efecto de los POT se postergue frente a inmuebles que aún tienen licencias aprobadas incluso con normas anteriores a la entrada en vigencia de la ley de reforma urbana, mediante la figura de los Planes Urbanísticos Generales. Hay temas normativos que no viene al caso contar, pero hay una necesidad de controlar el carrusel de las licencias, también en las curadurías cuando una curaduría niega la licencia.

En cuanto al control de las construcciones, se solicitó la supervisión técnica e interventoría externa. Igualmente, se pidió que el certificado del permiso de ocupación de la obra, expedido por el Supervisor Técnico, fuera un requisito para la escrituración del inmueble. Además, se exigió que se expidiera la constancia del cumplimiento de las obligaciones urbanísticas por parte del constructor y el anexo de la bitácora de obra, porque aunque se dice en la ley que su entrega al administrador y a la autoridad urbana es obligatoria, al solicitarla al constructor, muchos de ellos prefieren no entregarla o entregar una reformada. Esto pasa día a día en los edificios.

Por último, en el tema de las sanciones, se solicitó actualizar la ley 66 de 1968 que todavía regula las sanciones por incumplimiento en las obligaciones como enajenador de vivienda, que no es una infracción urbanística, con multas de $\$ 5.000$, \$50.000 y $\$ 100.000$. Adicionalmente, se pidió agregar como causal de sanción la ocupación de los inmuebles sin el certificado de permiso de ocupación, el incumplimiento en la entrega jurídica y material de las áreas objeto de cesión gratuita y el incumplimiento de los deberes y las sanciones expresadas en las licencias. 
Se espera un compromiso por parte del Ministerio de Vivienda para sacar adelante esta ley, en la que ellos adicionaron como escenario la creación del seguro obligatorio para la Vivienda Segura

\section{Ruta Medellín Vivienda SEgurA 2014-2024}

Luego de analizar con profundidad el colapso de la torre 6 de la urbanización Space, más los otros daños presentados en las edificaciones construidas por el grupo CDO, y que hoy son objeto de análisis por parte de la Universidad de los Andes en el estudio encomendado por la Alcaldía para determinar las causas del colapso y el estado de la estructura de los edificios que quedan en pie, el Alcalde de Medellín, Aníbal Gaviria Correa, determinó construir una ruta que se denominó Ruta Medellín Vivienda Segura 2014-2024. En el Foro Urbano Mundial realizado en abril de 2014, se decía que ojalá en diez años la gente fuera a nuestra ciudad a estudiar la seguridad de las edificaciones, para crear así un método de reparación integral al daño que se le hizo a la ciudad. Esta ruta la vamos a realizar en compañía de la Cámara Colombiana de la Construcción (Camacol). Con este proyecto buscamos tres objetivos: 1. Garantizar que los afectados por CDO puedan restablecer su patrimonio (de ahí surge la necesidad de crear un seguro de estabilidad de edificaciones); 2. Incorporar al nuevo Plan de Ordenamiento Territorial todo lo relacionado con seguridad de vivienda y de edificaciones y 3. Definir el esquema del control urbano más adecuado para el cumplimiento de la ley.

El proyecto estratégico le hará seguimiento a los procesos de control urbanístico y al cumplimiento de las normas y las obligaciones de las empresas constructoras, implementando medidas para revisar las licencias de construcción otorgadas por las curadurías municipales. Además, articulará acciones con las autoridades locales y nacionales con el fin de generar confianza en los compradores de vivienda, adoptando las normas que se consideren necesarias en el Plan de Ordenamiento municipal (acuerdos y decretos específicos) para que haya un mayor control por parte del municipio de Medellín a la construcción de vivienda.

Para terminar, todo el tema se resume en una sola palabra: ética. Aquí lo que faltó fue ética, sobró innovación pero realmente faltó mucha ética que es la que, de verdad, se debe aprender en las aulas de la universidad.

\section{REFERENCIAS}

Decreto 1469 de 2010 (30 de abril), por el cual se reglamentan las disposiciones relativas a las licencias urbanísticas; al reconocimiento de edificaciones; a la función pública que desempeñan los curadores urbanos y se expiden otras disposiciones. Diario Oficial No. 47.698.

Flórez, G. (16 de octubre de 2013). 'El desplome del edificio Space es un caso aislado’. Portafolio.

Jiménez, G. (5 de septiembre de 2014). Calculista de Space diseñó cuatro sedes de Bancolombia. El colombiano, recuperado de http://www. elcolombiano.com/calculista_de_ space_diseno_cuatro_sedes_de_ bancolombia-HGEC_309860

Ley 388 de 1997 (18 de julio), por la cual se modifica la Ley 9 de 1989, y la Ley 2 de 1991 y se dictan otras disposiciones. Diario Oficial No. 43.091.

Ley 400 de 1997 (19 de agosto), por el cual se adoptan normas sobre construcciones sismo resistentes. Diario Oficial No. 43.113.

Revisión de cálculos estructurales en el edificio Bancolombia de Rionegro. (26 de mayo de 2014). Periódico El Oriente, recuperado de http://periodicoeloriente.com/revision-de-calculos-estructurales-en-el-edificiobancolombia-de-rionegro/ 